\title{
Physical Investigation into an array of onshore OWCPs designed for water delivery
}

\author{
Davide Magagna $^{1, *}$, Dimitris Stagonas ${ }^{1}$, Gerald Muller $^{1}$ \\ ${ }^{1}$ Sustainable Energy Research Group, University of Southampton, Southampton, United Kingdom \\ * Corresponding author. Tel: +44(0) 238059465, Fax: +44 (0)23 8067 7519, E-mail:d.magagna@soton.ac.uk
}

\begin{abstract}
An OWC Wave Pump (OWCP) for seawater desalination is under development at University of Southampton. The paper presents experimental results for work carried out on an array of OCWPs at a scale of 1:40. The interaction between singles components of the array is determined in order to assess the layout which gives the maximum power output from an array of 3 OWCPs. The results provide a benchmark for comparison against the data available in literature obtained from BEM simulation. Results show that amplification of the wave signal up to 4.8 times can be achieved within the array. Increasing the distance between devices by two times the width of the chamber resulted in a reduction of the magnification factor up to $30 \%$.
\end{abstract}

Keywords: Arrays, Oscillating Water Column, Separation Distance, Capture width

\section{Nomenclature}

$M W L$ Mean Water Level

$N$

$O W C$ Number of devices in array

WEC .................... Wave Energy Converter

$q \quad$...................................Array Factor

A Section of duct ........................................ $\mathrm{m}^{2}$

$d_{s} \quad$ Separating distance .........................m

$g \quad$ Gravitational constat............................. $m \cdot \mathrm{s}^{-2}$

$H \quad$ Wave height .......................................... $m$

$h \quad$ Water depth.............................................. $m$

$l \quad$ Output duct length ................................... $m$

$l_{1} \quad$ Input duct length ....................................... $m$
$P_{a} \quad$ Power output of Array ......................

$P_{s} \quad$ Power output of a Single device .............. $\mathrm{kW}$

$P_{\text {out }}$ Power output.......................................... $\mathrm{kW}$

$Q \quad$ Flow rate ............................................ $\mathrm{m}^{3}$ :s

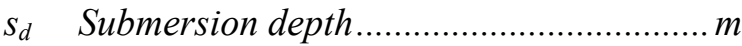

$T_{N} \quad$ Natural period of Oscillation ..................... $S$

$T_{W}$ Incoming wave period ........................... $s T$

$z_{r} \quad$ Removal height........................................

$\alpha \quad$ Angle of inclination of output duct........ rad

$\rho$ density.............................................. $\mathrm{kg} \cdot \mathrm{m}^{-3}$

$\omega_{\mathrm{D}} \quad$ Wave Frequency.............................. $\mathrm{rad}^{-1} \mathrm{~s}^{-1}$

$\omega_{\mathrm{N}} \quad$ Natural Frequency Oscillation.......... $\mathrm{rad} \cdot \mathrm{s}^{-1}$

\section{Introduction}

Recent progresses made on the development of Wave Energy Converters (WECs) have encouraged researchers to evaluate the deployment of arrays of WECs in order to maximize the power-output. Whereas it would seem straightforward that the output obtained from an array of WECs is higher than the power generated by multiple items working separately, the interferences between devices and waves could have a negative effect reducing the overall power output. The effects generated by the geometrical disposition of the device are measured by the $q$ factor, as presented by Babarit in [1]. $q$ represents the ratio between the power output $P_{a}$ generated by $N$ devices deployed in array configuration, against the power of $N$ devices working autonomously $P_{s}$, e.g without interaction.

$q=\frac{P_{a}}{N^{\prime} P_{s}}$

When $\mathrm{q} \geq 1$ positive effects are obtained by the array disposition of multiple WECs. One of the determining factors in the evaluation of $q$ is the separation distance, $d_{s}$, which indicates the 
space between two devices in the array. The practical role of $d_{s}$ is to influence the interaction between the radiated waves generated by the oscillation of each single device.

The determination of $q$, thus far, has been predominantly conducted using numerical phase resolving Models. Mathematical models are used to simulate the wave-device dynamics and to assess the performances of the array. They allow for a faster evaluation of the problem, but present limitations due to the formulation of the problem. Linearization of the equations involved, and assessment of infinitely long arrays being the main case. Alexandre et al [2] investigated the changes in the performances of point absorbers WEC disposed in array by assessing changes in the wave field due to the radiation of each components. Falcao presented the case of power extraction by a periodic linear array OWC (Oscillating Water Column) [3]. Other examples of mathematical model for the evaluation of WECs working in array can be found in [4-6]. The use of physical tests to evaluate the performances of arrays is, however, limited mostly due scaling problems and to the availability of appropriate facilities.

Current research at University of Southampton is focusing on the development of an Oscillating Water Column Wave Pump (OWCP) for water delivery. The device is designed to operate in arrays in order to maximize water delivery and increase the frequency response spectrum. This paper presents the results obtained from physical model tests carried on array of 3 OWCPs.

\section{The OWCP and Array Configuration}

The OWCP is a resonant type WEC, based on the more common Oscillating Water Column (OWC). The OWCP is designed to exploits the resonant conditions obtained during the oscillatory motion of the water contained in the chamber to deliver water to a fixed height. The OWCP can be considered as an overtopping type of WEC; however it differs from the standard overtopping devices such as the Wave Dragon [7] or the Composite Sea Wall [8], since they exploit the run-up of the water over an inclined ramp to deliver water to a reservoir.

The device is composed of two-part duct; with a horizontal underwater section (input duct), and an inclined pumping section extending above Mean Water Level (MWL) (Figure 1). The OWCP acts as a resonator with natural period of oscillation equal to $T_{N}$. To maximize performances the device has to be tuned with the incoming wave period $T_{W}$. It is possible to implement resonance control by varying the angle $\alpha$ of inclination of the output duct, e.g. changing the mass of water contained within the OWCP. 


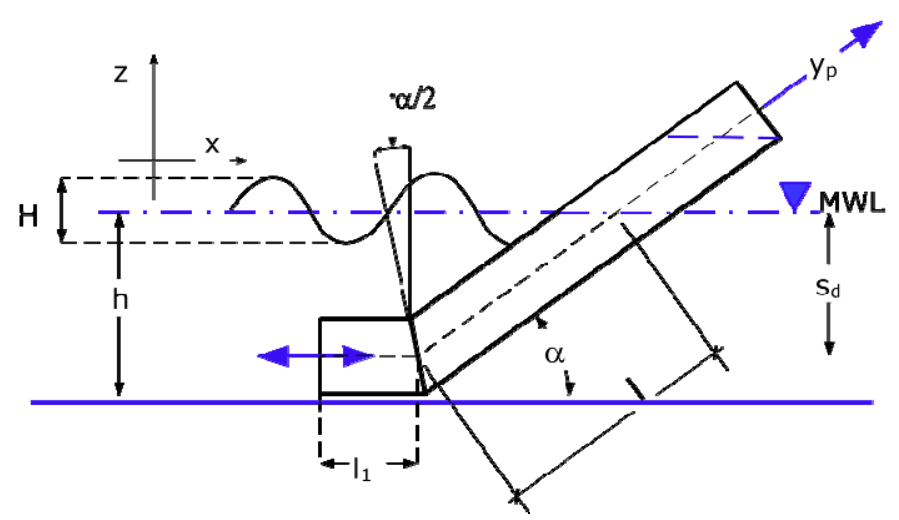

Figure 1. Schematic definition of the OWCP WEC. Where lis the length of the output duct, $l_{1}$ is the input duct, $y_{p}$ the delivery height of water, $h$ water depth, $H$ is the wave height, $s_{d}$ the submersion depth and $\alpha$ is the angle of inclination.

In order to maximize the delivery of water, the deployment of arrays of multiple OWCPs has been considered. Arrays of 3 OWCPs are considered in this paper. In particular, a close investigation focuses on the deployment of 3 differently tuned OWCPs devices in order to maximize performances and broaden the array response under different wave conditions. The concept of using differently tuned devices is justified by the need to provide a simple resonance control system for the array, and to phase out the destructive radiation waves generated by the downward motion of the column of water exiting the device. Initial results on the response of an array of multiple OWCPs have shown that the deployment of multiple devices broadens the frequency response of the array [9]. The role of the separation distance over the performance is therefore assessed.

\section{Methodology}

Experimental tests were carried in order to assess the performance of the different configuration of the arrays. The tests were carried in a $4 \mathrm{~m}$ long, $1.7 \mathrm{~m}$ wide and $0.4 \mathrm{~m}$ deep wave basin. Froude scale was employed with a scale factor $\Lambda=40$. Linear waves were generated by a piston type wave maker, with the wave heights ranging between $H=1-4.5$ $\mathrm{cm}$ and period $T_{W}$ between 0.8 and $2 \mathrm{~s}$. The water depth in the basin was kept at $14 \mathrm{~cm}$, with submersion depth $s_{d}$ of $7 \mathrm{~cm}$. 7 models of the OWCP were built out of transparent acrylic (3 $\mathrm{mm}$ thick). Their characteristics are presented in Table 1.

Table 1. Specifications of the models of the OWCP built for 1:40 scale tests.

\begin{tabular}{cccccc}
\hline Model & Inlet shape & $\begin{array}{c}\text { Dimension } \\
(\mathrm{mm})\end{array}$ & $\alpha$ & $\begin{array}{c}l_{l} \\
(\mathrm{~mm})\end{array}$ & $\begin{array}{c}T_{N} \\
\left(s_{d}=7 \mathrm{~cm}\right)\end{array}$ \\
\hline OWCPS1 & Square & $24 \times 20$ & $30^{\circ}$ & 40 & $0.851 \mathrm{~s}$ \\
OWCPS2 & Square & $24 \times 20$ & $30^{\circ}$ & 40 & $0.851 \mathrm{~s}$ \\
OWCPS3 & Square & $24 \times 20$ & $30^{\circ}$ & 40 & $0.851 \mathrm{~s}$ \\
OWCP20 & Circle & $24 \varnothing$ & $20^{\circ}$ & 55 & $1.022 \mathrm{~s}$ \\
OWCP25 & Circle & $24 \varnothing$ & $25^{\circ}$ & 50 & $0.935 \mathrm{~s}$ \\
OWCP30 & Circle & $24 \varnothing$ & $30^{\circ}$ & 40 & $0.851 \mathrm{~s}$ \\
OWCP35 & Circle & $24 \varnothing$ & $35^{\circ}$ & 35 & $0.795 \mathrm{~s}$ \\
\hline
\end{tabular}

The configurations of the array tested are presented in Table 2, along with the separation distances between the devices. 
Table 2. Configurations of the type of arrays tested. The * indicates the device located in the centre of the array. The Square array employs 3 similarly tuned devices.

\begin{tabular}{ccc}
\hline Array name & Models used & Separation distances $(\mathrm{mm})$ \\
\hline Square & OWCPS1- OWCPS2*- OWCPS3 & $0-15-30 \mathrm{~mm}$ \\
$20-25-30$ & OWCP20- OWCP25*- OWCP30 & $0-30-60 \mathrm{~mm}$ \\
$20-25-30 \mathrm{~W}$ & OWCP20- OWCP25*- OWCP30 & $0-30-60 \mathrm{~mm}$ with reflective wall \\
$25-30-35$ & OWCP25- OWCP30*- OWCP35 & $0-15-30 \mathrm{~mm}$ \\
\hline
\end{tabular}

The arrays are located in the centre of the wave basin, with an absorbing beach installed on the back to reduce the reflection of waves from the walls (Figure 2). Resistance type wave gauges are employed to monitor the wave conditions. Wave Gauges are also installed within each device in the array to determine the lift of water (Figure 3).

The performances of the array and of each device are assessed with the magnification factor, $M$, given by equation (2).

$M=\frac{y_{p}}{H}$

Where $y_{p}$ represents the lift of water within each OWCP. By using $M$ to assess the performances of the arrays, the case of no Power Take Off (PTO) installed is evaluated. The power output, $P_{\text {out }}$, of each device can be estimated in relation with to $M$ or $y_{p}$, once the delivery height $z_{r}$ is defined, as shown in equation(3). This relates to the crest Power determined by Margheritini et al. [10] for the assessment of the efficiency of the SSG wave energy device.

$P_{\text {out }}=Q z_{r} r g$

Where $Q$ represents the flow rate of the delivered water, $\rho$ the water density and $g$ the gravitational constant. For each incoming wave $Q$ can be determined by

$Q=\left(y_{p} \sin a-z_{r}\right)^{\prime} \frac{A}{T_{W}}$

Where $A$ represents the cross-sectional area of the OWCP duct. For the study presented in this paper the value of $Q$ can be estimated over a wave cycle. For irregular waves, the average $Q$ has to be determined. It has to be noted that both $Q$ and $A$ are both frequency dependent, therefore maximum values of $P_{\text {out }}$ can only be achieved close to resonance conditions. 


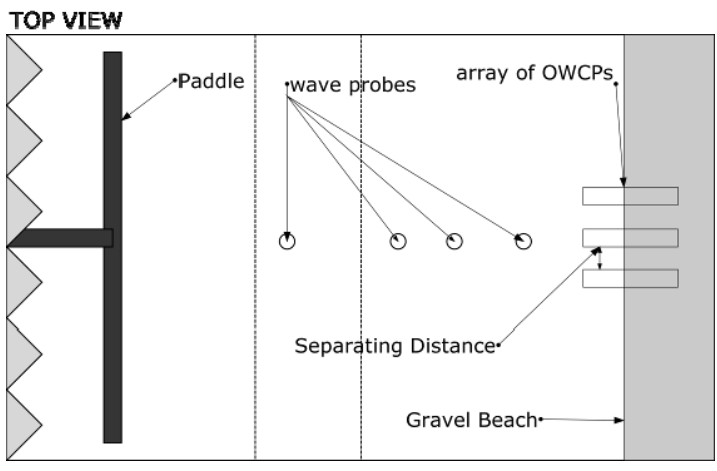

Figure 2. Experimental Setup

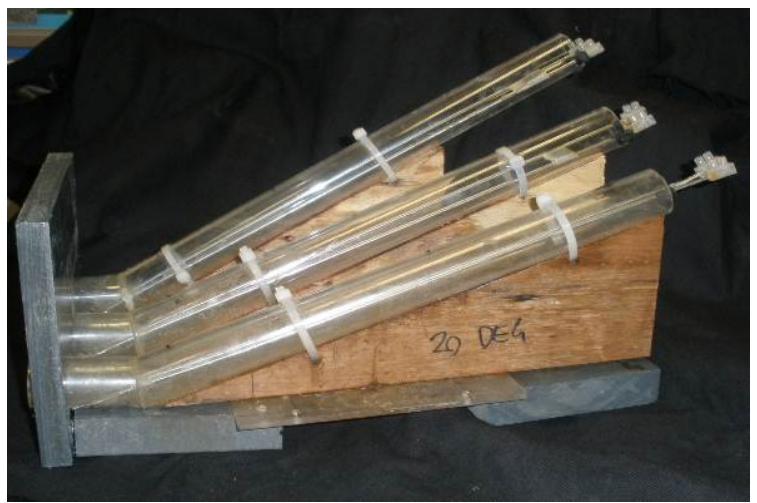

Figure 3. Array of 3 OWCP devices

\section{Results}

The response of each component of the arrays is assessed, and the values of $M$ for different wave conditions is then determined. These are compared in order to determine the effects of the separation distance on the single and overall performance. Figure 4 presents the changes in $M$ with $d_{s}$, for the 25-30-35, Square and 20-25-30 arrays configuration. It can be noticed that positive effects towards the delivery in the central pipe are achieved in each array configuration. It can be seen that for the cases when the devices are differently tuned higher values of $M$ are achieved. With increasing separating distances, the values of $M$ drop. On average a reduction in $M$ of $14 \%$ is seen by increasing the $d_{s}$ from 0 to $15 \mathrm{~mm}$, with a further reduction of $12 \%$ moving from 15 to $30 \mathrm{~mm} d_{s}$. Only the OWCP25 (Figure 4.a.) and OWCP20 (Figure 4.b) devices are subject to an increase in $M$ with $d_{s}$. In Figure 5 the changes of $M$ for different $d_{s}$ are assessed along with the changes in the non-dimensional frequency
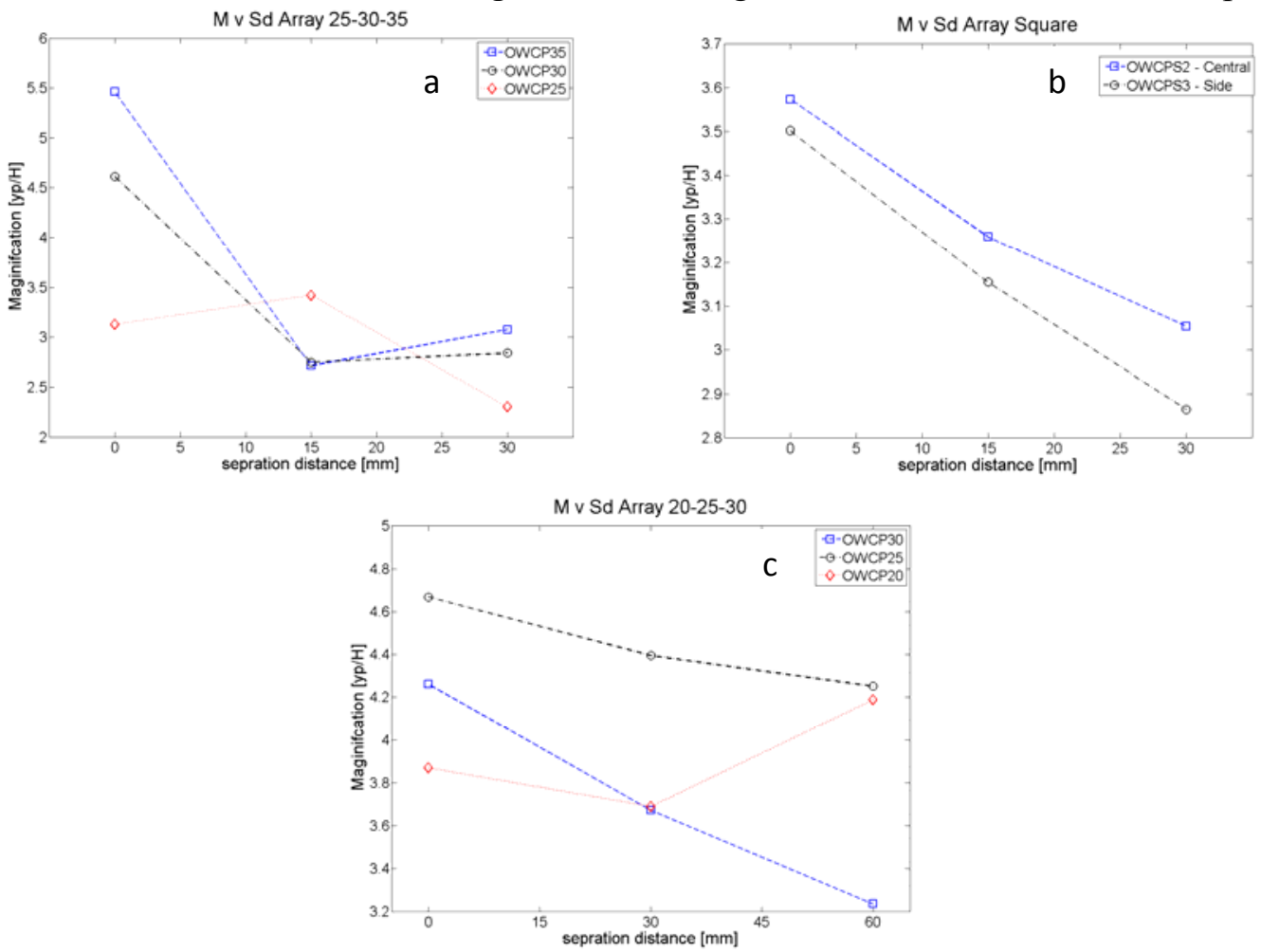

Figure 4.a) Changes in $M$ with $d_{s}$ for the 25-30-35 Array configuration. b) Changes in $M$ with $d_{s}$ for the Square Array configuration. c). Changes in M with $d_{s}$ for the 20-25-30 Array configuration. 


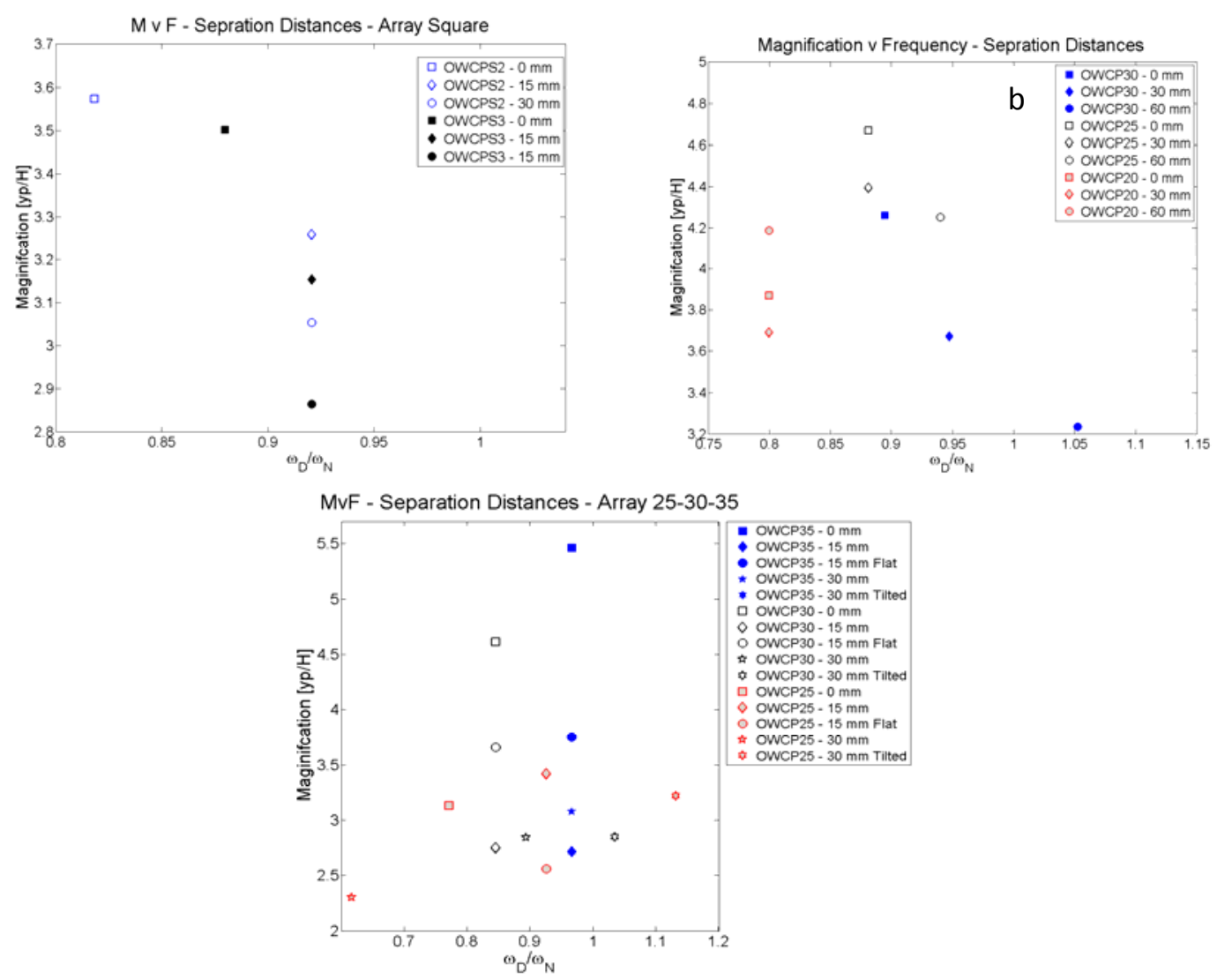

Figure 5. Maximum M against the non dimensional frequency $\omega_{D} / \omega_{N}$, for different $d_{s}$. a) Square Array. b) 20-25-30 Array. c) 25-30-35 Array. The Flat configuration considers the mouth of the device being leveled, whilst Tilted indicates the central pipe being pushed forward compared to side devices.

$\omega_{D} / \omega_{N}$. The non-dimensional frequency represents the ratio between the angular wave frequency $\omega_{D}$ and the natural frequency of oscillation of the device $\omega_{N}$. When the ratio is close to 1 , each device is operating as a stand-alone and no interferences are affecting the performances of the device. It can be seen that, with the increase in $d_{s}$, all devices tend to operate as stand alone, with maximum $M$ achieved when $\omega_{D} / \omega_{N}=1$.

Maximum delivery however are achieved for $d_{s}=0 \mathrm{~mm}$ with $\omega_{D} / \omega_{N} \cong 0.8$. It is believed that strong arrays interference affects the damping of the devices, causing as a result a stronger response, hence higher $M$ are obtained. Even for the cases presented in Figure 5.b and Figure 5.c different behaviors are observed in the OWCP20 and OWCP25 device. In the first case changes in $M$ and $d_{s}$ do not reflect changes in $\omega_{D} / \omega_{N}$, whilst for the OWCP25 a wider spectrum of frequencies is obtained.

Figure 6 and Figure 7 present the responses, expressed in terms of $M$, of the central and side device for the Square Array and for the 20-25-30 Array respectively. In both cases it can be seen how higher $M, 3.57$ and 4.67 respectively, are achieved in the central OWCP. Figure 6 and Figure 7 show the effect of $\omega_{\mathrm{D}} / \omega_{\mathrm{N}}$ and of the wave steepness on $M$, it can be noticed that the area of response of the devices broadens with minimum separating distance. In Figure 6, where results for a Square Array are presented, one can notice that both devices present a similar response, however the central device presents a broader amplification area compared to the OWCPS3 located on the side. In Figure 7, it is possible to notice how the bandwidth response reduces for both the OWCP30 and OCWP25 with increasing $d_{s}$. Furthermore, a steady decrease of $M$ can be noticed in both devices, indicating negative interaction between 
devices.
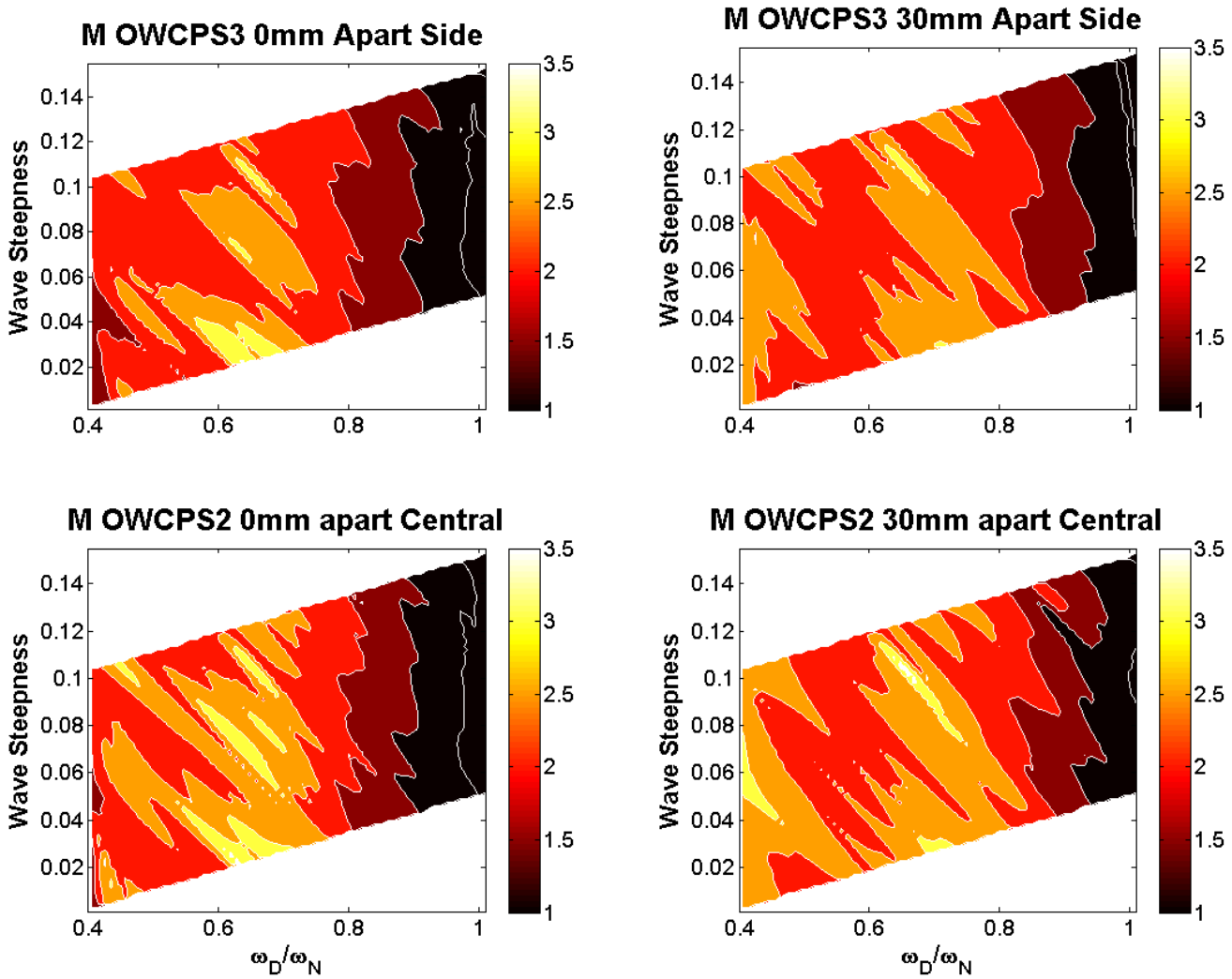

Figure 6. Magnification Factor for the components of the Square Array. Focus in given at the behavior of the side pipe (OWCPS3, top) and at the central pipe (OWCPS2, bottom) for values of $s_{d}=0$ and $30 \mathrm{~mm}$ (left and right).
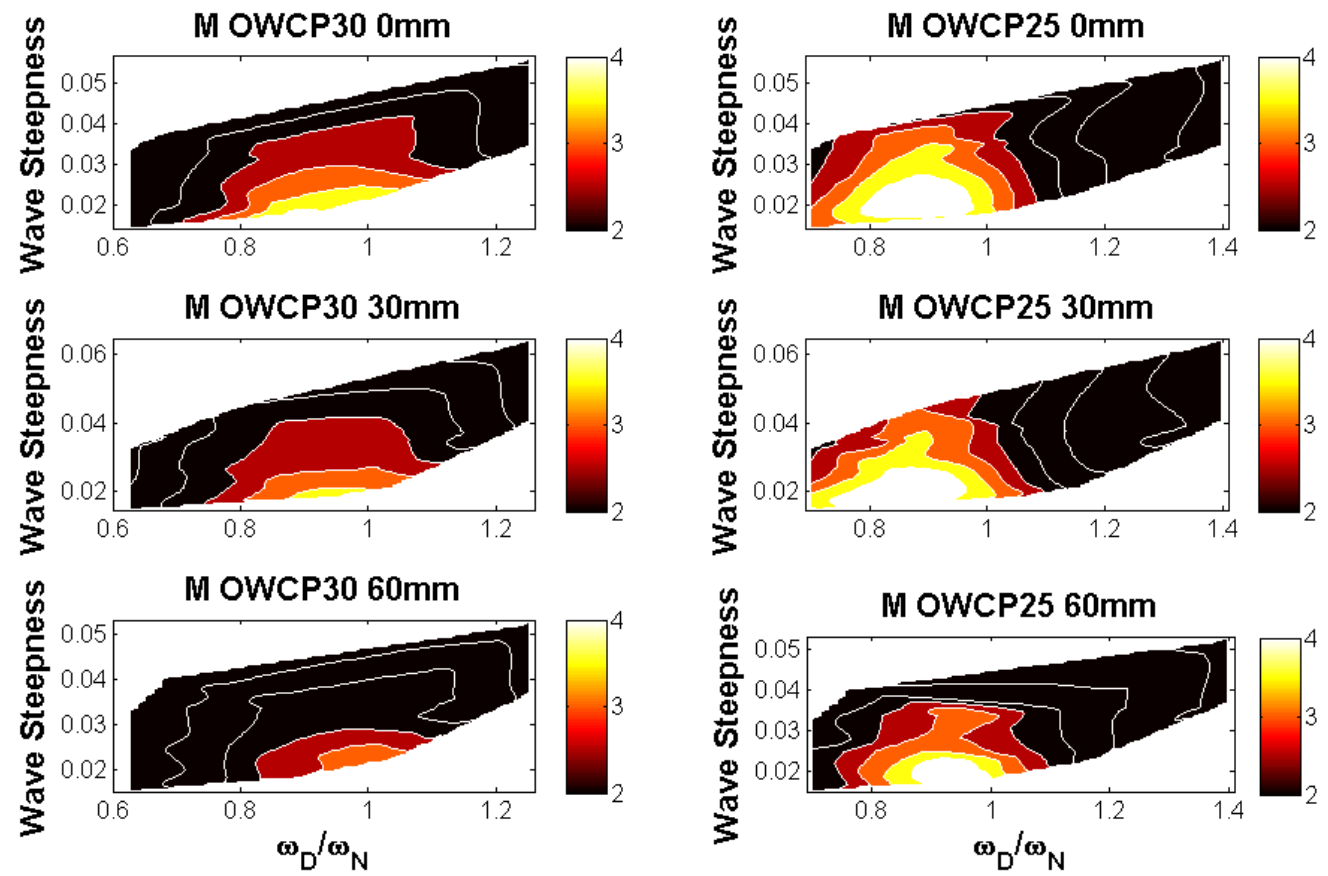

Figure 7 Magnification Factor for the components of the 20-25-30 Array. Focus in given at the behavior of the side pipe (OWCP30, left) and at the central pipe (OWCP25, right) for values of $s_{d}=0$, 30 and $60 \mathrm{~mm}$ (top to bottom.). 


\section{Conclusions}

In this paper physical experiments on arrays of onshore OWCP devices have been presented. The main aim was to investigate the role of the separating distances between devices, and how it affected the overall performance of the device and the one of the array. Array installations for WECs have been considered in order to amplify the power output of a single device.

In this paper arrays of similarly tuned devices, as well as differently tuned devices have been investigated. From experimental testing it has been highlighted that when the devices are operating with a minimum separating distance, better performances. In particular, the device located in the centre is positively affected in all the cases investigated.

The results presented show better performances by the arrays with differently tuned devices. Maximum values of $M$ were obtained for the cases when $d_{s}=0$. The values of $M$ varied according to the array configuration with $M=5.46$ for the 25-30-35 array, $M=4.669$ for the 20-25-30 array and $M=3.573$ for the square array. Reduction in $M$ of $14 \%$ can be expected by increasing $s_{d}$ of $15 \mathrm{~mm}$, however the reduction is dependent on the configuration. Decrease in $M$ varied between $30 \%$ for the $25-30-25$ array to $6 \%$ for the $20-25-30$ for a $30 \mathrm{~mm}$ increase in the separating distance. The results obtained show that $s_{d}$ and configuration of the device play a strong role on affecting the performances of the arrays.

This is believed to be due to the different phase responses by the water column exiting the device in the downward motion. In the downward motion the mass of water generates a radiated wave that contrasts the incoming wave train interfering with the energy conversion process in the OWCP. When the devices in the array are phased out, the radiation is minimized and higher $M$ can be achieved. The same can be assumed for $15-30 \mathrm{~mm} d_{s}$, when the devices are separated the radiated waves affects the operation of the nearer devices.

The work here presented shows that it is possible to increase the bandwidth response of multiple devices by arranging them in array configurations. The overall performances, however, are dependent on the separation distance between the devices and their natural period of oscillation. It has been shown that by reducing to a minimum the distance between the devices, maximum performances can be achieved.

\section{Acknowledgements}

The research leading to these results has received funding from the European Community's Seventh Framework Programme (FP7/2007-2013) under grant agreement n ${ }^{\circ} 212423$.

\section{References}

[1] A. Babarit, Impact of long separating distances on the energy production of two interacting wave energy converters. Ocean Engineering, 2010. 37(8-9): p. 718-729.

[2] A. Alexandre, T. Stallard, and P.K. Stansby. Wave field modification due to a WEC array. in Coastlab - Third International Conference on the Application of Physical Modelling to Port and Coastal Protection. 2010. Barcelona, Spain: Grupo Pacifico.

[3] A.F. Falcão, Wave-power absorption by a periodic linear array of oscillating water columns. Ocean Engineering, 2002. 29(10): p. 1163-1186. 
[4] M. Folley and T.J.T. Whittaker, The effect of sub-optimal control and the spectral wave climate on the performance of wave energy converter arrays. Applied Ocean Research, 2009. 31(4): p. 260-266.

[5] P. McIver, Wave interaction with arrays of structures. Applied Ocean Research, 2002. 24(3): p. 121-126.

[6] P.C. Vicente, et al., Dynamics of arrays of floating point-absorber wave energy converters with inter-body and bottom slack-mooring connections. Applied Ocean Research, 2009. 31(4): p. 267-281.

[7] J.P. Kofoed, et al., Prototype testing of the wave energy converter wave dragon. Renewable Energy, 2006. 31(2): p. 181-189.

[8] G. Muller, et al. Composite Seawalls for Wave Energy Conversion. in Coasts, Marine Structures and Breakwaters 2009 2009. Edinburgh, Scotland, United Kingdom: ICE.

[9] D. Magagna, et al. Physical investigations into the capture width of an array of OWC Wave Pumps for maximum efficiency. in 3rd International Conference on Ocean Energy. 2010. Bilbao, Spain: ICOE

[10] L. Margheritini, D. Vicinanza, and P. Frigaard, SSG wave energy converter: Design, reliability and hydraulic performance of an innovative overtopping device. Renewable Energy, 2009. 34(5): p. 1371-1380. 